\title{
The Semantic Analysis of the Preposition "Through" From the Perspective of Cognitive Linguistics
}

\author{
Fang Yue \\ School of Foreign Languages, Dalian University of Technology, Dalian, China \\ Shuo Cao* \\ School of Foreign Languages, Dalian University of Technology, Dalian, China \\ Jing Huang \\ School of Foreign Languages, Dalian University of Technology, Dalian, China
}

\begin{abstract}
Prepositions are the most active words in English. Since the late 20th century, linguists have made some deep and comprehensive studies about the polysemy of prepositions. Especially, the emergency of cognitive linguistics offers a new perspective for the study of prepositions. Under the framework of cognitive linguistics, this paper will analyze the preposition "through" from six domains by means of cognitive theories, including prototype theory, image schema theory and conceptual metaphor. It is found that the prototypical meaning of "through" is its spatial meaning. And the extension of the word is achieved with the aid of source-path-goal schema and spatial metaphor from the spatial domain to non-spatial domains. Based on these findings, a conclusion is drawn that all the non-prototypical meanings of one particular preposition are derived from its prototypical meaning and the study of the prototypical meaning is of great significance to the acquisition of English preposition meaning.
\end{abstract}

Index Terms - through, cognitive linguistics, prototype theory, image schema, conceptual metaphor

\section{INTRODUCTION}

The development of cognitive linguistics in recent years provides a new perspective for the semantic study of prepositions.

Language is the necessary tool for people in the society to communicate ideas, express feelings and exchange information. Meaning, as the core of language, is the most important element. Only when people know the meaning of a word or a sentence, can they understand what people of that language want to express. So knowing the meaning of words in one particular language is one of the most essential steps to acquire that language (Cheng, 2012), but the difficulty is that one specific word may have several different meanings. When people see an English word, they are less likely to think of all the meanings of that word. As a result, it is necessary for linguists to find a convenient way to acquire the word meaning.

From the cognitive perspective, language is one of the elements of human cognition. There is a close relationship between language and human cognition. On the one hand, cognition provides the basis for the development of language. Throughout the history, the evolution of cognition precedes that of language. Language is the product of cognition developing at a certain stage. The premise of language expression is that people have already had some knowledge of the word. On the other hand, language can facilitate the development of cognition and consolidate the outcome of cognition. The emergency of language has a positive influence on human cognition. For one thing, language is of great help for perceiving new things. For another, language is an essential tool for people to communicate thoughts, exchange information, and enhance the cognitive capacity.

Cognitive linguistics provides the possibility to probe into word meaning and reveals the nature of mental vocabulary from a totally novel perspective. It is believed that different meanings of the same word are projected into our mind by means of image schema, not semantic features or abstract prepositions. Surrounding a primary sense, a semantic or polysemy network is formed. And the extension of meaning is achieved under the help of cognitive theories including prototype theory, image schema theory and conceptual metaphor. Based on all the cognitive theories mentioned above, this paper will make a detailed cognitive analysis about different meanings of the polysemy "through".

In this paper, a considerable illustration of the cognitive theories will be made, including the prototype theory, the image schema theory and the conceptual metaphor before making a cognitive analysis of the preposition through to

* Shuo Cao is the corresponding author, Email: caoshuo@dlut.edu.cn 
discuss the prototypical meaning, its spatial meaning and its metaphorical meanings to dig out the relationship between its prototypical meaning and non-prototypical meanings.

\section{LITERATURE REVIEW}

Prepositions are the most active words in English and they can collocate with nouns, verbs, adjectives and adverbs to formulate hundreds of thousands of collocations. Most of the prepositions are polysemes. One preposition even has more than ten meanings and, in most cases, people do not learn all the meanings of that word at a time. Therefore, if people are able to learn prepositions in a systematic way, they can have the ability to acquire all the meanings of the same preposition.

\section{A. Previous Studies on Polysemy and Acquisition of Prepositions}

Although prepositions only occupy a small proportion in English vocabulary, they are frequently used. In both Brown and LOB corpora, prepositions account for $12 \%$ (Mind \& Weber, 1989). According to Kennedy (2000), on average, there is one preposition for every eight words in any English text. The original meaning of many prepositions is used to express spatial position and spatial relations, such as "in", "out", "down", "up" and so on. In addition to their spatial meanings, English spatial prepositions also have rich metaphorical meanings. So the seemingly simple prepositions have become difficult for English learners.

Previous studies on prepositions mainly laid emphasis on the following two general types. One is from the perspective of polysemia of prepositions (Cao, 2005; Nie, 2001). Those linguists mainly analyze the meaning of prepositions from the theoretical level by combining the prototype theory and spatial metaphor theory. Another type focuses on the acquisition of English spatial prepositions (He, 2010; Li, 2008; Ma, 2007). These studies analyze the extension of English preposition and the influence of native language thinking on English learners through the comparisons of English and Chinese preposition meaning. Some mainly lay emphasis on the contrastive analysis of prepositions in English and Chinese. Our learning process of prepositions in English is often interrupted by our knowledge of native language. Actually, the meaning of the preposition in Chinese is relatively indistinct and ambiguous, while the meaning of the preposition in English is varied and subtle. Therefore, the usage of preposition in Chinese cannot be copied into that in English.

\section{B. Previous Studies on Semantic Connection between Meanings of Prepositions}

Previous studies on English prepositions also paid much attention on the semantic study of English prepositions and the connection between their meanings. For example, Vandeloise (1994) discusses the meaning of the preposition "in" and the connection between its meanings. Tyler \& Evans (2001) systematically studies the internal structure of the meaning of the preposition "over".

Until recent years, linguists have attached their attention to the analysis of prepositions from the cognitive approach. Some linguists apply cognitive linguistics to the teaching of English prepositions to explore the influence of this theory on the semantic acquisition of prepositions, such as Tyler \& Evans (2004), who take "over" as an example to study the application of cognitive linguistics to the semantic acquisition of prepositions. Previous studies focus on prepositions like "in", "on" and "over". The study of "through", however, is not well-researched. The present paper aims to analysis the preposition "through" from the cognitive perspective to study its metaphorical and non-spatial meanings.

\section{THEORITICAL FRAMEWORK}

In order to have a better semantic analysis of the preposition "through", it is necessary to obtain some knowledge about cognitive theories, especially prototype theory, image schema theory, conceptual metaphor theory.

\section{A. Prototype Theory}

Prototype theory is a new theory of semantic analysis and now is applied to various areas of cognitive science. It is used to describe the nature at first, and then is employed to cognitive linguistics and cognitive semantics.

It is a theory of word meaning according to which meanings are identified, in part at least, by characteristic instances of whatever class of objects, etc. Since "prototype" is the most representative and typical member in a category, it can be used as a cognitive reference point for other members in the category (Wang, 2006). For example, people think of songbirds, such as a robin, as having more of the central character of a bird than others, such as ducks, falcons, or ostriches. In that sense, a robin or the like is a prototypical instance, or prototype, of a bird. But birds are the denotation of bird. Prototype theories are often linked with the view that denotations have no precise limits. For example, bats are merely less "robin-like" than ducks and so on. A notion of prototype has also been applied to other aspects of language.

The study of prototype theory in the west is much earlier than that in our country. This theory is proposed by Rosch in the 1970s. According to Rosch, things that can be defined as prototypes have three characteristics: 1) perceptual salience, that is, they are easy to distinguish. 2) memorability, that is, they are easy to remember. 3) stimulus generalization, that is, one can think of other objects with a physical-like function according to the features of the prototype.

Prototype effect is a cognitive process that is ubiquitous in daily life. People understand the world through the 
prototype classification, which is to understand things and their relationship with the world based on the similarity between objects or structures and typical members of the category they belong to. This means that there is always a recognized core or typical member in every category, and people can know less typical or peripheral members of that category by understanding this core or typical member. Koveceses (2002) points out that archetypes not only exist in everyday concepts, but also in language structures. That is to say, prototype effects can not only occur on concrete objects in the objective world, but also on abstract concepts and ideas represented by language structures.

Many researchers, experts and professors in the field of cognitive linguistics prototype and categorize English words according to the prototype theory. The polysemous phenomenon of English prepositions is a further expansion of the detailed archetypal connotation, so as to enhance the expression ability of English prepositions. According to some experts, the prototype of English words are continuously adapting to different needs of new expression through social development. The fundamental principle lies in that, on the basis of the basic connotation, English prepositions can be endowed with other metaphorical meanings not beyond the family resemblance.

\section{B. Image Schema Theory}

In recent years, cognitive linguistics has been a new perspective to the study of language. Cognitive linguists believe that human's cognition has a great influence on language. As one of the most important parts of cognitive linguistics, image scheme has increasingly become the focus of people's attention. It endows the ability for human beings to perceive the world to pursue the nature of people's cognition system.

Researchers of cognitive semantics, Lakoff and Johnson (1990), first proposed the notion of image schema. Johnson (1990) holds that image schema is a recurring dynamic pattern, providing stability and structure to our experience. Lakoff (1990) puts forward the Idealized Cognitive Model (ICM) in his work "Woman, Fire and Dangerous Things" to give further explanation of this theory. Lakoff (1990) believes that image schema is the simple experience relationship and spatial orientational relations that constantly appear in daily experience, such as up-down, front-back, part-whole, center-periphery, etc. These basic relationships and structures constitute the framework and cornerstone of human cognition of the world (Lakoff, 1990).

Therefore, people generally define image schema as a type of basic conceptual structure identified in cognitive linguistics. Schemata such as Path and Container are held to be prelinguistic structures based on bodily experience and help to shape the form of linguistic categories. They are used to describe, for example, the semantics of spatial, temporal and aspectual expressions (Zhang, 2011).

Lakoff (1987) emphasizes the dynamic features of image schema. He believes that the formation of the image schema is mainly shaped by sensorimotor. Therefore, kinesthetic sense is the key method for us to experience the world. According to him, image schema can be classified into the following types: containment schema, part-whole schema, link schema, center-periphery schema and path schema, etc.

Taking containment schema as an example, containment schema derives from our experience of the human body itself as a container; from experience of being physically located ourselves within bounded locations like rooms, beds, etc; and also of putting objects into containers. Lakoff and Johnson (1900) identify CONTAINER as one of a group of ontological metaphors, where our experience of non-physical phenomena is described in terms of simple physical objects like substances and containers.

The physiological basis of the source-path-goal schema is that when an object moves from one point to another, it has a starting point, an ending point and a path. The basic logic is that when the object runs from the starting point to the ending point along the path, it must go across every point on the path. The further you go down the path, the more time you are going to spend.

Image schema is widely used in the study of polysemy, especially in the study of prepositions. In the process of word meaning expansion, concrete image schemata can be formed through physical experience. And these concrete image schemata form more abstract image schemata by means of the expansion and transformation of metaphor and metonymy mechanisms. Therefore, the metaphorical expansion of lexical meaning can be formed. Different meanings of the same preposition can be demonstrated by different schemata.

From the above analysis, image schema theory plays a great role in language researches. When explaining some linguistics phenomena, traditional linguistics lacks sufficient explanatory power and persuasion. Image schema provides the cognitive basis and the source of experience for language research. It is a powerful tool for the study and explanation of some linguistic phenomena.

\section{Metaphor Theory}

Aristotle, as the forefather of metaphor, has a positive impact on the study of metaphor. In the western classical rhetoric, Aristotle is the first people to make a comprehensive learning of metaphor. He mentioned this notion many times in his works, especially in his classics Poetics and Rhetoric. What's more, he also gives a definition and makes some classification of metaphor. Since Aristotle's time, metaphor has been a heated topic for scholars in the west.

Aristotle believes that metaphor is the modification of language. And, for most of us, metaphor is a figure of speech in which one thing is compared to another by saying that one is the other, as in "Tom is made of iron". Here, it would probably say that the word Iron is used metaphorically to communicate eloquently, to impress others with beautiful, aesthetically pleasing words, or to express some deep emotion. Perhaps it should also add that what makes the 
metaphorical identification of Tom with Iron possible is that Tom and Iron have something in common, namely, their perseverance and persistence.

Under the motivation of the development of modern cognitive linguistics, our understanding of metaphor has already changed from a traditional rhetorical device to a way of cognition. And on the basis of cognitive linguistics, conceptual metaphor is first proposed by Lakoff and Johnson (1980). They believe that metaphors are ubiquitous in everyday life, not only in language, but also in our thought and activities. And the conceptual system that our thought and activities rely on is metaphorical in nature (Lakoff \& Johnson, 1980). Conceptual metaphor holds that metaphor is a systematic projection mapping from a concrete concept to an abstract one. The former is the source domain and the latter is the target domain. People tend to understand abstract, unfamiliar, complex and intangible concepts by means of concrete, familiar, simple and tangible concepts. Metaphor is a question of thought, which can be reflected in language. It is a kind of way of thinking and cognitive method. Lakoff and Johnson (1980) classify conceptual metaphor into three types: ontological metaphor, structural metaphor and orientational metaphor. This notion overturns the traditional metaphor which is merely used as a rhetorical device.

In the cognitive linguistic view, metaphor is defined as understanding one conceptual domain in terms of another conceptual domain. A convenient shorthand way of capturing this view of metaphor is that conceptual domain (A) is conceptual domain (B), which is what is called a conceptual metaphor. A conceptual metaphor consists of two conceptual domains, in which one domain (the target domain) is understood in terms of another (the source domain). A conceptual domain is any coherent organization of experience. Conceptual metaphor is based on culture and experience. Therefore, it is different in different cultures.

Generally speaking, "space" and "metaphor" are usually treated and studied as two notions in different categories. As a matter of fact, "space" and "metaphor" are not without any connection. They have already been associated with each other since very early time. Metaphor is an important tool for people to perceive the world. And spatial metaphor is a metaphor of image schema, that is, spatial concept is used as the source domain and other non-spatial concepts are the target domain. Abstract notions must be constructed by spatial metaphor; therefore, spatial metaphor plays an essential role in human's cognitive activities.

Spatial metaphor adequately shows the laws that people perceive the world. The perceiving of the spatial concept is the foundation of the cognition of the physical world, whereas the perceiving of the time concept and other concepts are based on the cognition of the space (Dong, 2013). People can directly take advantage of their eyes and ears to perceive the space; therefore, they are able to use the spatial concept to describe the abstract notions like time, range, state, quantity and social status.

Taking the orientational metaphor as an example. It is one of the conceptual metaphors that enable speakers to make a set of target concepts coherent by means of some basic human spatial orientations, such as up-down, in-out, center-periphery, etc. In this type of metaphor, the orientational words are usually used as vehicle. For example, the expression "happy is up, sad is down", up-down can demonstrate distinct social and cultural experience, that is, the word "up" is usually associated with positive things, whereas "down" is in connection with bad things.

Spatial metaphor is best reflected in the semantic expansion of polysemous prepositions. Preposition is one of the common parts of speech in English. Its meaning and usage are complicated and changeable. The birth of spatial metaphor theory provides a new perspective for researchers engaged in the semantic research of English prepositions. The archetypal meaning of a space preposition indicates the static position or the movement path of an object in space. With metaphor as the cognitive mechanism, the concept of space can be mapped to other abstract concepts, so that words can generate other metaphorical meanings on the basis of their original meanings.

In this study, the author will take advantage of the prototype theory to investigate the prototypical spatial meaning of the preposition "through" and then to study how the meanings of through can be extended to non-spatial domains from the spatial domain with the aid of image schema theory and spatial metaphor.

\section{A Cognitive ANALYSIS OF "Through"}

Having knowing knowledge of some cognitive theories, this part will make a detailed analysis of the preposition "through" from the perspective of cognitive linguistics to study its prototypical meaning and non-prototypical meaning and the relationship between them.

\section{A. Prototypical Meaning of "Through"}

Prototype is the term used in semantics and psycholinguistics, for a typical member of the extension of a referring expression. In other words, it is the semantic information that is more prominent or typical than other information among various semantic items of the same word. From this point of view, all the meanings of a preposition are closely related to a principled method to a prototype.

It is widely believed that the earliest meaning of a particular preposition is probably its prototypical meaning. And the earliest meaning of a preposition is usually the first entry of all the meanings of this word (Wu, 2017). "Earliest attested meaning can be gotten from the dictionary." (Xiong, 2008, p.40) According to this view, it can make an assumption that the prototypical meaning originates from the earliest meaning while the non-prototypical meaning from the prototypical meaning. 
So after having looked up several dictionaries, the author obtains the prototypical meaning of through. The first entry of through in the Werriam-Webster's Advanced Learner's English Dictionary (2009) is "into one side and out of the other side of (something). And the earliest meaning of through in Collins Cobuild Essential English Dictionary (1998) is "to move through a hole or opening means to move directly one side or end of it to the other." And "moving in one side and out of the other side of (an opening channel, or location) in New Oxford American Dictionary (1989). Therefore, the prototypical meaning of through denotes a spatial meaning that TR (Trajectory) traverses a bounded LM (Landmark) with an entrance point and an exit point.

\section{B. Spatial Meaning of "Through"}

Through the study of the prototypical meaning of through, the author concludes that its prototypical meaning or its original meaning is its spatial meaning. Therefore, spatial meaning is worth to study.

\section{Image Schema of "Through"}

In cognitive linguistics, different image schemata are described by means of the position and the movement of TR (Trajectory) and LM (Landmark). TR is the asymmetric subject and its spatial direction is to be determined. LM is the reference substance. The way that TR goes across is called Path (Hu, 2008). After having detailed analysis of the image schema theory, it can be known that the image schema of through belongs to source-path-goal schema. This kind of schema consists of three elements: source (the starting point), goal (the destination) and path (the way connecting the starting and the ending point). When LM and TR are stable and at rest, Path is zero.

\section{Static and Dynamic Meaning of "Through"}

When TR is relatively still in a certain point or an area of LM, the meaning of through is static. And when TR is continuously moving towards an end point across LM, the meaning of through is dynamic. Here the author provides some examples to illustrate this meaning.

1) An atmosphere of anticipation vibrated through the crowd.

2) The security guards pushed their way through the door.

3) They spent a couple of hours walking through the mall.

In the first sentence, through demonstrates a static sense and has a meaning of "over all the parts of (something)". In this sentence, TR is "an atmosphere of anticipation". It is the subject of the action. LM is "the crowd" ---a solid space. In this case, LM and TR are entirely overlapped.

In the second sentence, through indicates a dynamic sense and has a meaning of "from one side or end to another side or end of (something). In this sentence, TR is "the security guards". LM is "the door". The Path is the way he walks toward the door. In the third sentence, the preposition is used to describe movement within a place or an area of land, air, etc. In this sentence "they" (TR) walked to various places in the mall (LM).

\section{Metaphorical Meaning of "Through"}

In addition to the basic spatial meaning of through, there are also some metaphorical meanings extended from its original meaning. In this part, metaphorical meanings of through will be analyzed from five domains.

\section{From Spatial Domain to Temporal Domain}

The conceptualization of space and time is different. The concept of space can be directly experienced by people, whereas the concept of time is defined by means of metaphor and is comparatively abstract. Time can be conceptualized as a bounded space, and a thing or a time period that takes place or is experienced can be seen as a certain point or a part of that bounded space. At the same time, the meaning of through is expanded and has a meaning of "from the beginning to the end of a period of time".

1) He slept through the movie.

2) Many students work through the summer.

3) We left halfway through (= in the middle of) the lecture.

In the sentences above, TRs are "he", "many students" and "we" respectively, LMs are "the movie", "the summer" and "the lecture". TR touches LM and goes across it. In these sentences, "through the movie", "through the summer", and "through the lecture" are developed from the basic spatial concept ---"from one side or end to the other side or end". Their meanings are metaphorized and their image schemata are the same as "through the tunnel" in people's mind. In this way, the spatial concept is projected to the temporal concept. Thus, the preposition "through" has an additional meaning in temporal domain. That is to say, "through" in these sentences indicates the movement of time. The difference is that TR only moves at the middle of LM in the sentence three.

What's more, this preposition can be used to indicate the numbers, days, etc., that are included in a range and has the sense of "until and including".

1) The store is open Monday through Friday.

2) Read chapters 2 through 5 for homework.

Therefore, the two sentences actually mean that the store is open Monday, Tuesday, Wednesday, Thursday, Friday, and read chapter 2, 3, 4 and 5 respectively. 


\section{From Spatial Domain to Domain of Cause and Reason}

According to the spatial image schema of the preposition "through", TR moves from one side or end to the other side or end along the path. In this process, the state of TR may be totally changed due to the action of external forces. If this spatial image is projected onto concrete things, it reflects a causal relationship. In this case, through means "because of or due to", which shows the relationship between a state or situation and a particular process.

1) The company's profits increased through improved sales.

2) We will succeed only through (= by means of) hard work.

3) Knowledge that is gained through life experience.

In sentence 1, the verb "increase" describes the process of "the company's profits" (TR) changing from "low" to "high" because of the "improved sales" (LM). TRs of the following two sentences are "we" and "knowledge" respectively, their states also change as a result of the forces of "hard work" and "life experience".

\section{From Spatial Domain to Domain of Manner}

In many cases, path can be seen as a method to achieve some purpose, fulfill some tasks and practice certain activity. Thus, the meaning of "by means of" is added to the preposition.

1) The leaders communicate through interpreters.

2) I learned of the job opening through her.

3) They ruled the country through fear.

Sentence 1, only if they have the interpreters (LM), the leaders (TR) can communicate. TR provides some assistance to the communication and TR likes a bridge to connect two leaders. In the second sentence, "I" knew of the job mainly thanks to "her" (LM). Also, the last sentence, they (TR) ruled the country under the assistance of fear (making people afraid)

\section{From Spatial Domain to State Domain}

The spatial concept of through "from one side to the other side" can be projected onto the state field. TR comes into contact with LM and goes across it. This process projects onto concrete event and generates the meaning of completion and produces the meaning of "in the process of (something)" or "experiencing (something)"

1) We are through the worst part of the storm.

2) I think she will be more comfortable at college not that she is through her first term.

The first sentence, "we" is TR, and "the storm" is LM. In this sentence, "we", motivated by "storm", are moving from one side of the storm to the other side of the storm, that is from the beginning of the storm to the end of the storm. Thus, the sentence means the worst part of the storm has ended. In the second sentence, TR is "she" and LM is "semester". The sentence means she has completed her first semester and produces the meaning of "experiencing the first semester" or "in the process of the first semester".

\section{From Spatial Domain to Sensory Domain}

In our experience of the physical world, people obtain the cognition and sensory meanings through the sensory organs, like eyes, ears, nose, mouth and our body. And those sensory meanings are also the essential parts of our sources of information. Using the spatial concept to understand abstract sensory information is also a research area of conceptual metaphor theory. Mapping from the spatial domain to the sensory domain indicates that TR overcomes certain sensory barrier to get certain sensory information.

Most of the information is acquired from the sense of sight. Therefore, the image schema in this mood is that TR overcomes obstacles for the sense of sight to achieve certain visual information.

1) She could see a figure through the fog.

2) I looked through the window.

3) She looked through the binoculars.

In the sentences above, TRs are people and LMs are "fog", "window" and "binocular" respectively. In order to get visual information, LMs that the sense of sight can go across are somewhat transparent. Not only the visual information, but the auditory information and some other sensory information can be obtained under the help of image schema between TR and LM in the sensory domain mapped from the relationship between TR and LM in the spatial domain.

In this part, the author analyzes the prototypical meaning of through. The prototypical meaning of through is its spatial meaning that TR traverses a bounded LM with an entrance point and an exit point. The image schema of through is source-path-goal schema. This preposition has both the static meanings and dynamic meanings, which can be demonstrated clearly by different image schemata. Then the author carries out the metaphorical extension of through. The non-prototypical meaning can be obtained through the extension of the typical meaning (the prototypical meaning). The meaning of through can be extended from the spatial domain to the temporal domain, the domain of cause and reason, the domain of manner, the domain of state and the sensory domain. And the author illustrates the various non-prototypical meanings of through with examples.

\section{CONCLUSION}


From the cognitive point of view, detailed analysis of the preposition "through" has been made.

First, the author looks into the prototypical meaning of through by analyzing the meanings of it in different dictionaries. The prototypical meaning, in most cases, is its earliest meaning or the first entry in the dictionary. So the prototypical meaning of through is its spatial meaning, that is, moving in one side or end and out of the other side or end of (something). It is the meaning that people think of at first when others mention it.

Second, the image schema of through is well illustrated. The meaning of it is a kind of source-path-goal schema. It contains a static meaning, i.e. over the parts of (something). That is to say, TR is stable in a certain point or an area in the LM. Certainly, it also has a static meaning and a dynamic meaning, i.e. from one side or end to the other side or end of (something). In other words, TR is moving towards the other side or end of LM.

Third, metaphorical meanings of through are analyzed in detail. Meanings of through in the spatial domain can be extended to other domains. Followings are its metaphorical extensions: "from the beginning to the end of a period of time" and "until and including" in the time domain; "because of" in the domain of cause and reason; "by means of" in the domain of manner; "in the process of (something)" or "experiencing (something)" in the state domain. In addition to these meanings, when TR overcomes certain sensory barrier, the sensory information can be gotten. All of these metaphorical extensions derive from the prototypical meaning of the preposition.

Although the author has found lots of meanings and examples in several dictionaries and made a relatively detailed study of them, there are still some limitations. For example, different meanings and examples looked up in the dictionaries are not adequate enough. Therefore, it is not able to make an entirely comprehensive investigation of through in spite of the scientific method the author used. Another point needed to be noticed is that the meaning of through may be in constant change when it collocates with other prepositions or adverbs, such as through with and all through.

Therefore, in order to comprehensively and deeply understand the metaphorical extensions of preposition "through", it is necessary to expand the research scope, increase the investigation items and make a longitudinal follow-up survey. This study not only provides a relatively satisfactory tool for exploring the cognitive semantic of "through", but also paves a way for studying words of other parts of speech in terms of cognitive perspective. Meanwhile, this paper also reminds English teachers as well as students to pay more attention to the meaning of "through" in a sentence, which makes the polysemes much easier to be learned.

\section{ACKNOWLEDGEMENTS}

This work was supported by the National Social Foundation of China (Grant Number 19BYY088).

\section{REFERENCES}

[1] Collins Cobuild Essential English Dictionary. (1998). China Translation and Publishing Corporation, 836-837

[2] Cheng, X. J. (2012). Semantic Analysis of Take from Cognitive Linguistic Perspective. Dalian Maritime University.

[3] Dong, S. S. (2013). A Cognitive Study of Through. Jiangsu University of Science and Technology.

[4] George Lakoff. Woman, Fire, and Dangerous Things. (1987). The University of Chicago Press.

[5] Hu, T. H. (2008). A Cognitive Analysis on Spatial Metaphor of Preposition "Through". Journal of Changchun University, 11(6), 46-48.

[6] Kovecses, Z. (2002). Metaphor: Metaphor. Oxford University Press.

[7] The Oxford English Dictionary. (1989). Oxford University Press.

[8] Webster's Third New International Dictionary. (1961). G\&C. Merriam Company, Publishers.

[9] Werriam-Webster's Advanced Learner's English Dictionary. (2009). Encyclopedia of China Publishing House, 1715

[10] Wu, J. (2017). Semantic Analysis on the Preposition "Through" from the Cognitive Perspective. Journal of Chengdu Normal University, 33(7), 65-70.

[11] Wu, W. S. (2011). Cognitive Linguistics and Chinese Studies. Shanghai, Fudan University Press.

[12] Wang, Y. (2006). Cognitive Linguistics. Shanghai Foreign Language Education Press.

[13] Zhang, X. (2011). The Semantic Analysis of the Preposition "Through". Journal of Hubei Adult Education Institute, 17(5), 81-82.

Fang Yue was born in Shandong, China on January 10, 1999. She obtained a bachelor's degree from Qingdao University of Science and Technology and is studying for a master's degree in cognitive linguistics and psycholinguistics in Dalian University of Technology.

Shuo Cao, a professor in Dalian University of Technology. Her research interest includes cognitive linguistics, psycholinguistics, cross-cultural communication, second language acquisition and translation.

Jing Huang was born in Shandong, China. She obtained a bachelor's degree from Qufu Normal University and is studying for a master's degree in cognitive linguistics and psycholinguistics in Dalian University of Technology. Her research interest is visual metaphor. 